\title{
Choice from Non-Choice: Predicting Consumer Preferences from Blood Oxygenation Level-Dependent Signals Obtained during Passive Viewing
}

\author{
Ifat Levy, ${ }^{1,2 *}$ Stephanie C. Lazzaro, ${ }^{3 *}$ Robb B. Rutledge, ${ }^{3}$ and Paul W. Glimcher ${ }^{3,4,5}$ \\ ${ }^{1}$ Section of Comparative Medicine and ${ }^{2}$ Department of Neurobiology, Yale University School of Medicine, New Haven, Connecticut 06520 , and ${ }^{3}$ Center for \\ Neural Science, ${ }^{4}$ Department of Psychology, and ${ }^{5}$ Department of Economics, New York University, New York, New York 10003
}

Decision-making is often viewed as a two-stage process, where subjective values are first assigned to each option and then the option of the highest value is selected. Converging evidence suggests that these subjective values are represented in the striatum and medial prefrontal cortex (MPFC). A separate line of evidence suggests that activation in the same areas represents the values of rewards even when choice is not required, as in classical conditioning tasks. However, it is unclear whether the same neural mechanism is engaged in both cases. To address this question we measured brain activation with functional magnetic resonance imaging while human subjects passively viewed individual consumer goods. We then sampled activation from predefined regions of interest and used it to predict subsequent choices between the same items made outside of the scanner. Our results show that activation in the striatum and MPFC in the absence of choice predicts subsequent choices, suggesting that these brain areas represent value in a similar manner whether or not choice is required.

\section{Introduction}

The choice process is typically viewed as a two-stage mechanism in which values are first assigned to each option and then compared to yield choice (Glimcher, 2009; Kable and Glimcher, 2009). Converging evidence suggests that these values are stored in the striatum and medial prefrontal cortex (MPFC), and are subsequently used by circuits in the lateral prefrontal and parietal cortices to guide choice (Kable and Glimcher, 2009). A separate line of evidence suggests that activity in these areas represents the values of rewards, even when choice is not required, as in classical conditioning tasks (O'Doherty, 2004; Knutson et al., 2005; Tobler et al., 2006). While traditional economic theory would partition these sets of findings (Samuelson, 1938), psychological models of valuation and choice would suggest that a common mechanism underlies these two sets of observations (Schultz, 2009). Does a single neural mechanism encode the value of options both when a choice between different options is required and in the absence of choice?

The first step toward answering this question was taken by Knutson et al. (2007). In their functional magnetic resonance imaging (fMRI) experiment, subjects viewed a consumer good,

\footnotetext{
Received June 21, 2010; revised Sept. 8, 2010; accepted 0ct. 21, 2010.

This study was funded by a joint National Institute of Neurological Disorders and Stroke-National Institute of Mental Health Grant R01-NS054775 to P.W.G. We thank Daniel Burghart and Mark Dean for helpful comments, Samanta Shaw for assistance with running subjects, Grace Yeh for pilot work, and Keith Sanzenbach and the New York University Center for Brain Imaging for scanning assistance.

*I.L. and S.C.L. contributed equally to this work.

Correspondence should be addressed to Ifat Levy, Section of Comparative Medicine, Yale University School of Medicine, P.0. Box 208016, New Haven, CT 06520. E-mail: ifat.levy@yale.edu.

DOI:10.1523/JNEUROSCI.3214-10.2011

Copyright $\odot 2011$ the authors $\quad 0270-6474 / 11 / 310118-08 \$ 15.00 / 0$
}

then viewed its price, and then chose whether to purchase the good. Striatal activity during the product presentation and MPFC activity during the price presentation predicted subsequent purchase decisions. These activations, however, were taking place in the context of a choice: subjects knew they would have to choose whether to purchase the item, and made their decision just a few seconds after viewing the good and its price. While these results provided strong evidence for the representation of subjective value in the striatum and MPFC during the choice process, they leave open the possibility that a different neural mechanism is engaged in the representation of value when choice is not required.

Lebreton et al. (2009) extended this finding to test the independence of choice and non-choice valuations directly. In their study, subjects first viewed images of faces, houses, and paintings in the scanner, while rating either their pleasantness or their age, and then made choices about pairs of the same items outside of the scanner. Parts of the striatum and MPFC were more active during both rating tasks for images that were subsequently chosen as more pleasant, suggesting that some type of valuation processes may indeed automatically occur in these areas.

The choices subjects made outside of the scanner in the Lebreton study, however, assessed the visual pleasantness of the images: by choosing, subjects indicated which image in a pair they experienced as more pleasant, not which item they would like to have. Here we extend this finding to the economic domain of consequential consumer choice, by determining whether activity in the striatum and MPFC during passive viewing can be used to predict the consumer goods that a subject will later choose.

To address this question, we used a simple non-choice lottery task to independently localize value-related areas in the striatum 


\section{Localization of value areas}
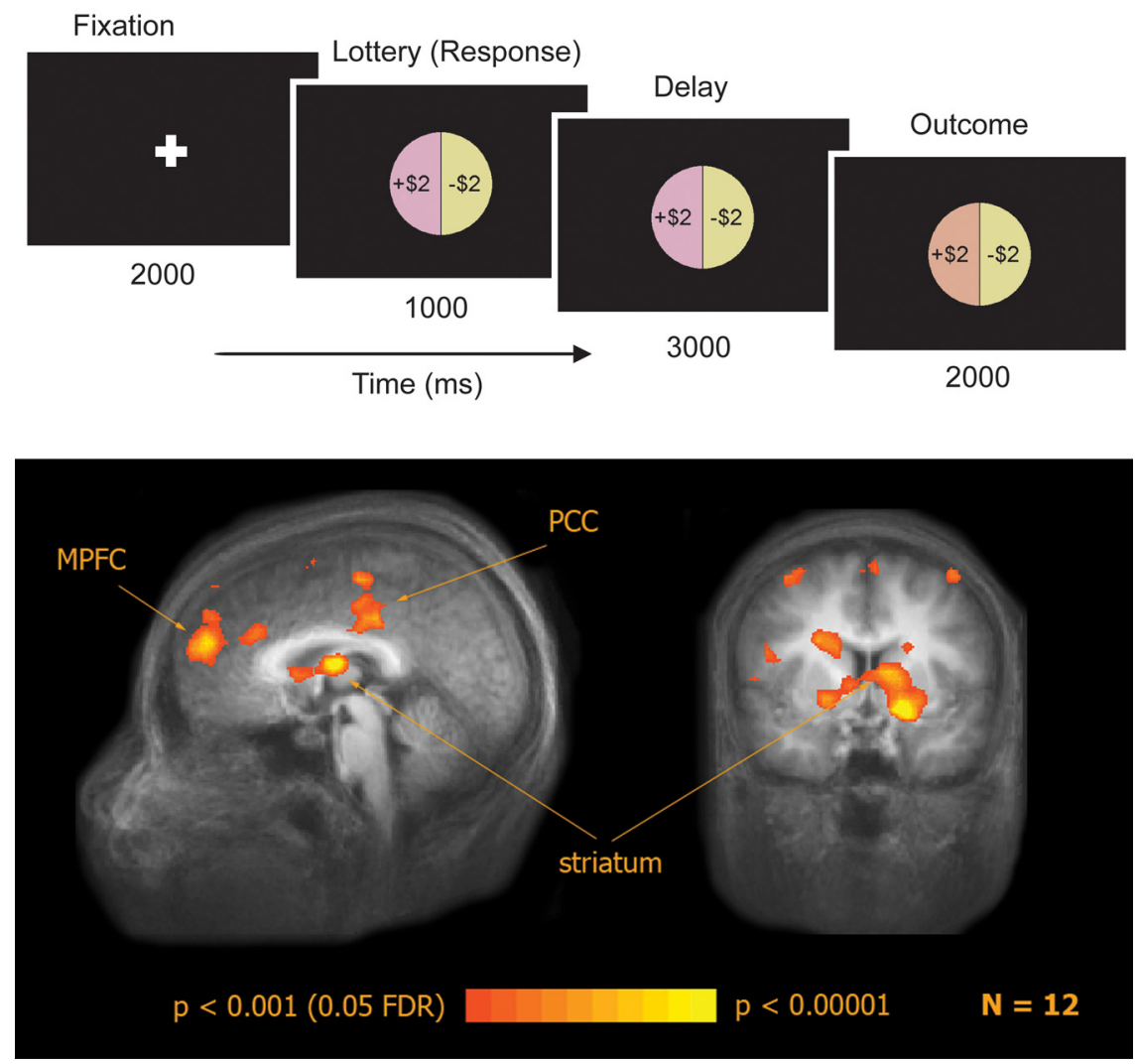

Figure 1. Localization of value-related areas with a functional localizer task: experimental design and activation in a win vs loss contrast.

and MPFC, where activation increases as value increases. Subjects then viewed images of individual goods in the scanner. Finally, outside of the scanner subjects made choices between all pairwise combinations of the same goods. If the striatum and MPFC represent the subjective value of consumer goods both during passive viewing of goods and during economic choice, then activation in these areas measured during passive viewing should allow us to predict subsequent choices. Furthermore, a wholebrain analysis using the preference rankings obtained outside of the scanner should also reveal significant activation in the striatum and MPFC.

\section{Materials and Methods}

Subjects

Twelve healthy right-handed adults ( 7 females; mean age, 26.33 years; age range, 18-34 years), with normal or corrected to normal vision participated in this study. All subjects gave written informed consent to participate and the experimental paradigm was approved by the University Committee on Activities Involving Human Subjects and was in compliance with the safety guidelines for MRI research. Subjects participated in two separate scanning sessions, one for the functional localizer and one for the goods task, and received a show-up fee of $\$ 25 / \mathrm{h}$ plus earnings based on the experiment, as detailed below. In each session, subjects were instructed in the task and tested for task comprehension before entering the scanner.

\section{Tasks}

Functional localizer task. To functionally localize value-related areas with as few prior assumptions as possible we used a non-choice task in which subjects win and lose money. This is similar to methods used to identify dopaminergic neurons used in electrophysiological experiments, which are based on the dopamine response to unexpected rewards (Schultz et al., 1997), and assumes that a value-related area will exhibit a response that increases as value increases. The experimental design was similar to the one developed by Caplin et al. (2010), except that only a single lottery appeared on each trial and subjects were not required to make any choices. Immediately before the scanning session, subjects received an endowment of $\$ 40$ that they were instructed to put in their pocket as they would be playing a lottery "game" with this money. The subjects were told that if they won more money over the course of the game, they would be given those winnings when the scan ended. If they lost any money during the game, they would have to return it to the experimenter and could keep the rest of the $\$ 40$. If at any point during the game they lost all $\$ 40$, the game would end and they would have to return all of the money. Each subject completed 128 trials of $8 \mathrm{~s}$ each in 2 scans. Each trial began with a $2 \mathrm{~s}$ fixation cross. Then a lottery represented by a pie chart appeared for $1 \mathrm{~s}$. The lottery was always equal probabilities of winning or losing $\$ 2$. To keep subjects alert, subjects were instructed to press a button during the $1 \mathrm{~s}$ lottery presentation. Following the button press, there was a $3 \mathrm{~s}$ delay period in which the lottery remained on the screen. Next, the outcome of the lottery was revealed for $2 \mathrm{~s}$ by a change in color of that outcome in the pie chart (Fig. 1). If subjects did not press a button within the $1 \mathrm{~s}$ time limit, the trial ended and the subject received a penalty of losing $\$ 2$.

Goods task. Subjects completed six scans of the goods task, each consisting of 41 trials. The first trial of each scan simply presented an image of a consumer DVD movie cover. This trial was used to capture the initial burst of activation at the beginning of a scan and all data from each of these first trials were discarded. In each of the next 40 trials presented during each scan, subjects passively viewed an image of one of 20 different items including four DVD movies (Pan's Labyrinth, Madagascar, Dodgeball, and Dreamgirls), two books (A Thousand Splendid Suns by Khaled Hosseini and The Road by Cormac McCarthy), four art posters (paintings by Monet, Lileger, Klimt, and Dali), three music CDs (Konvicted by Akon, Beethoven's Last Night by Trans-Siberian Orchestra, and Come Away With Me by Norah Jones), two pieces of stationery (a calendar/planner and a Moleskine notebook), and five monetary lotteries represented by pie charts. Each lottery offered a $50 \%$ chance of receiving a designated amount of money $(\$ 10, \$ 15, \$ 20, \$ 25, \$ 30)$ and a $50 \%$ chance of receiving $\$ 0$. Consumer goods were chosen based on a pilot study which showed that the ranking of these objects by members of our subject pool was highly idiosyncratic (no subject who participated in the pilot study participated in the experiment presented here). All items were presented 12 times in a random order to each subject. Each item was presented for $2 \mathrm{~s}$ followed by a fixation dot for $2 \mathrm{~s}$ (see Fig. $3 a$ ). Subjects were instructed that when they saw an item they should think about how much it was worth to them in a dollar amount. To keep subjects alert, on 20 random trials (one of the 12 presentations for each of the 20 items), after the $2 \mathrm{~s}$ fixation, subjects were asked whether they preferred the item they had just seen or a random amount of money (ranging from $\$ 1$ to $\$ 10$ ). The response had to be made within $1.5 \mathrm{~s}$, and was followed for $0.5 \mathrm{~s}$ by feedback; either the word "item" or "money" depending on their selection. If the subject did not respond within the $1.5 \mathrm{~s}$, the feedback "no response" was presented for $0.5 \mathrm{~s}$. Subjects were told that one of these question trials would be randomly selected at the end and they would receive their selection on that trial - the item or the money. Importantly, these 20 question trials were used only to maintain subject alertness and 
were excluded from all further analysis. On all of the 220 other trials, the fixation period was followed by the presentation of an " $X$ " for $2 \mathrm{~s}$, signaling that no question would be asked on that trial. All trials were followed by an $8 \mathrm{~s}$ period during which a fixation dot was presented at the center of the visual display. Importantly, during the scanning session subjects did not know that they would subsequently be asked to choose between the same items.

Behavioral choice task. Following the scanning session, subjects were removed from the scanner and asked to perform a choice task on a computer. Subjects made choices between all possible pairs of the items (see Fig. $3 b$ ). Options were presented in random order and subjects made a choice twice for each pair of items, with the items left-right flipped, for a total of 380 choices. Subjects were told that one random choice trial would be selected and they would receive whichever item they chose, so they should pick the item that they "really want" on every trial. Following the behavioral choice task, subjects completed a survey in which they saw each item and answered whether or not they knew what the item was, and whether or not they already owned the item.

\section{Imaging}

We used a 3-Tesla MRI scanner (Siemens Allegra head-only scanner) to measure changes in blood oxygenation level-dependent (BOLD) activity. During each $\mathrm{fMRI}$ scan, a time series of volumes was acquired using a T2*-weighted echoplanar imaging pulse sequence (repetition time, 2000 $\mathrm{ms}$; echo time, $30 \mathrm{~ms}$; flip angle, $75^{\circ}$; thirty-six $3 \mathrm{~mm}$ slices with no interslice gap; in-plane resolution, $3 \times 3 \mathrm{~mm}^{2}$; field of view, $192 \mathrm{~mm}$ ). Images were acquired using a custom radio frequency coil (NM-011 transmit head coil; Nova Medical Inc.). In addition, T1-weighted highresolution $\left(1 \times 1 \times 1 \mathrm{~mm}^{3}\right)$ anatomical images were acquired with a magnetization-prepared rapid-acquisition gradient echo pulse sequence, and used for volume-based statistical analysis. To minimize head movement, subjects' heads were stabilized with foam padding. Stimuli were projected onto a screen at the back of the scanner, and subjects viewed them through a mirror attached to the head coil.

\section{Data analysis}

Behavior. Subjects made all possible pairwise choices between the items they saw in the scanner. Each choice pair was presented twice, resulting in 380 choices in total. A preference ranking of the 20 items was computed for each subject based on the total number of times each item was chosen by that subject. Thus an item which was chosen every time, against all other possible prizes, would have been chosen 38 times and would necessarily have a preference rank of 1 . An item which was never chosen during the 38 rounds in which it was offered would have a rank of 20.

fMRI. fMRI data were analyzed with the BrainVoyager QX software package (Brain Innovation) and with additional in-house software written in Matlab (MathWorks Inc.). Preprocessing of functional scans included discarding the first 2 volumes, slice scan time correction, interand intrasession three-dimensional motion correction and removal of low frequencies up to 5 cycles per scan (linear trend removal and high pass filtering). The images were then coregistered with each subject's high resolution anatomical scan, rotated into the anterior commissureposterior commissure plane, and normalized into Talairach space (Talairach and Tournoux, 1988). For the multisubject analysis, the images were also spatially smoothed using an $8 \mathrm{~mm}$ full-width halfmaximum Gaussian filter.

Statistical maps. Statistical analysis was based on a general linear model (Friston et al., 1995). The time course of activity of each voxel was modeled as a sustained response during each trial, convolved with a standard estimate of the hemodynamic impulse response function (Boynton et al., 1996). For the functional localizer, the model included a predictor for "win" outcomes and a predictor for "loss" outcomes. The model was independently fit to each voxel activity time course, yielding two coefficients, one for wins and one for losses. The maps in Figure 1 highlight voxels in which the win coefficient was significantly larger than the loss coefficient in a multisubject random-effects analysis. For the goods task the model included a dummy predictor for the first trial of each scan, a dummy predictor for the presentation of each item and a parametric predictor of the number of times each item was chosen in the choice task outside of the scanner, normalized to a $0-1$ range. The model was independently fit to each voxel activity time course yielding three coefficients for each voxel. The maps in supplemental Figure 2 (available at www.jneurosci.org as supplemental material) highlight voxels in which the coefficient of the parametric predictor was significantly larger than zero in a multisubject random-effects analysis. The threshold for the random-effects maps was set at $p<0.05$ corrected for false discovery rate (FDR) (Genovese et al., 2002) and a spatial extent of at least $100 \mathrm{~mm}^{3}$.

Region of interest analysis. The functional localizer was used to define regions of interest (ROIs) within each individual subject, based on significantly higher activation for wins compared to losses ( $p<0.05$ uncorrected, spatial extent $>100 \mathrm{~mm}^{3}$ ). ROIs were defined in the MPFC, striatum, and occipital cortex for each subject. Note that these ROIs are independent from the goods task and specific to each subject. Time series for the goods task were extracted from each ROI of each subject, and percentage signal change was computed in each time point compared to the mean of the first point of the trial and the last two points of the preceding ITI. Responses to repetitions of the same items (excluding question trials) were first averaged and the percentage signal change at time points 4 and 5 after cue onset $(8-10 \mathrm{~s}$ after onset) were then averaged together to yield the activation level used for the prediction of choice (see Fig. 4), allowing time for the hemodynamic response. For supplemental Figure 3 (available at www.jneurosci.org as supplemental material), these responses were normalized to a $0-1$ range within subjects and averaged across subjects.

Prediction of choice. To predict choices in an unbiased manner we used the BOLD responses to the items in the goods task that were sampled from ROIs defined by the functional localizer as described above. For each pair of items, activations for the two items were compared and the item that gave rise to a higher activation level was predicted to be chosen. Since there is no way to correctly predict indifference using this approach (i.e., when a subject chose item A over B on the first repetition of the pair and item B over A on the second repetition) we excluded those choice pairs from further analysis. We then compared our predictions with the subjects' actual choices to determine prediction accuracy. Prediction accuracies were also calculated separately for the subset of items that subjects did not own, but were familiar with, and for the subset of items that excluded the lotteries. Note that our use of the term "prediction" is different from its use in multivariate studies (Haynes and Rees, 2006), where the term specifically refers to the use of an independent set of data to test the predictive power of pattern classifiers.

Finally, we separately calculated the prediction accuracy for pairs of items based on their distance in order of neural activity ranking. After ordering the items from highest to lowest neural activity magnitude and then grouping the data by ordinal rank distance we calculated prediction accuracy for all of the subsets of neural distances in our dataset. For example, the largest distance in a given dataset would be 19: this would compare the item with the highest neural activity with the item having the lowest neural activity. The smallest distances would be the set of all sequentially ranked pairs of items. The prediction accuracy between pairs of items at all possible neural rank distances is plotted in Figure 5, in Results.

\section{Results}

To test whether valuation areas represent value in a similar manner during the choice process and in the absence of choice, we identified value-related areas using an independent localizer, sampled activations in these areas during viewing of goods in the absence of choice, and then used these activations to predict subsequent choices made outside of the scanner.

\section{Independent localization of valuation areas}

To localize value-related areas we used a simple lottery task that did not involve choice. Each trial started with the presentation of a lottery, signaling an equal chance of winning or losing $\$ 2$. Following a short delay period, the outcome was revealed (Fig. 1, top). A random-effects group analysis of the contrast between 


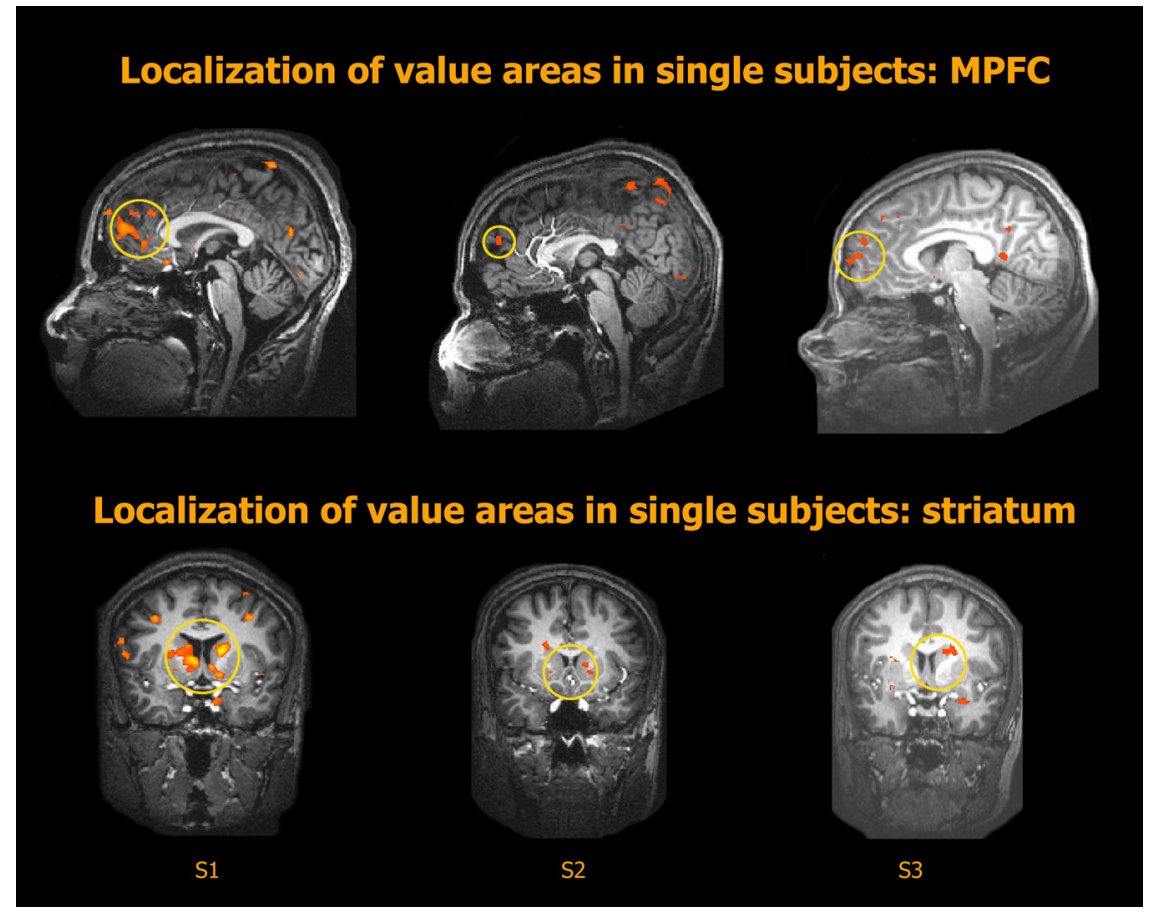

Figure 2. Localization of value-related areas with a functional localizer task in three example subjects (S1, S2, S3). Areas in the MPFC and the striatum that were significantly more active for wins than for losses in the functional localizer task were used as ROIs in the main experiment.

\section{a Viewing of goods in the scanner}

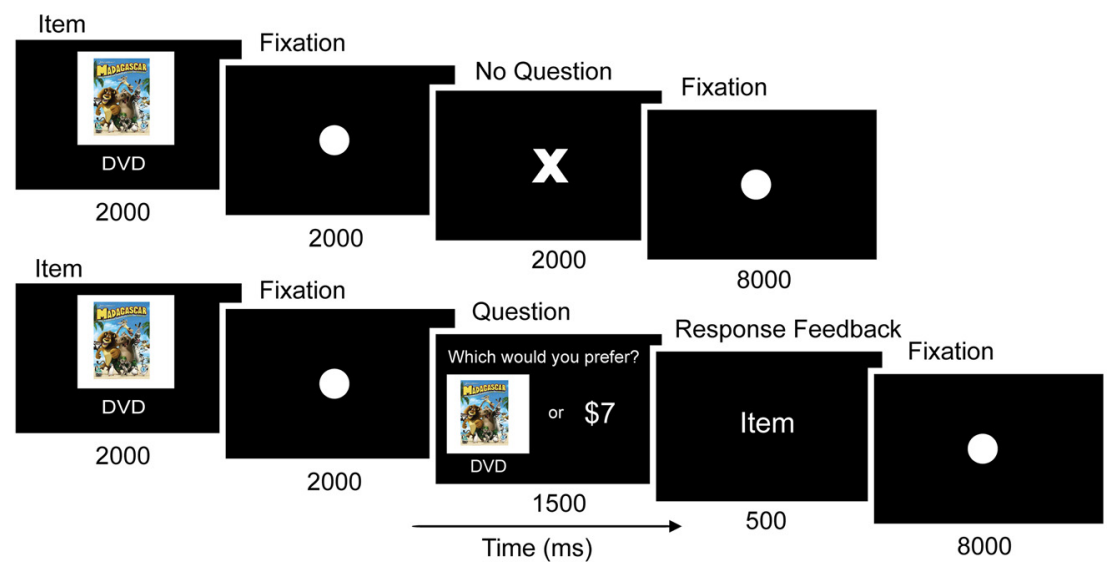

\section{b Actual choices outside scanner}

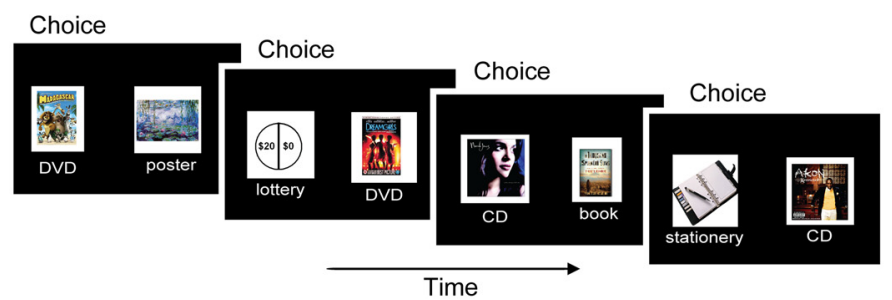

Figure 3. Experimental design for the goods task. $\boldsymbol{a}$, Passive viewing of items in the scanner (top). To maintain subjects' alertness, on a few random trials they were asked to choose between the item and a varied sum of money (bottom). These trials were not included in the analysis. $\boldsymbol{b}$, Outside of the scanner, subjects were asked to make pairwise choices between the same items that were presented to them in the scanner.

win and loss outcome trials $(n=12, p<0.05$, FDR corrected) revealed significant activation in the MPFC, the striatum, and the posterior cingulate cortex (PCC) (Fig. 1, bottom). In single subjects (Fig. 2) the most consistent results of the same contrast were found in the MPFC $(n=12, p<0.05$, uncorrected, spatial extent $>100 \mathrm{~mm}^{3}$ ) and the striatum $(n=11, p<0.05)$ and we therefore focused on these areas in subsequent stages of the analysis. Note that our localizer task was specifically designed to not distinguish between outcome values and reward prediction errors; these two quantities are perfectly correlated on each trial in our design. Therefore, some of the observed activation could have been specific to RPE rather than to value per se. We use the term "value-related areas" here in the broadest sense, to include any area whose activation is higher for higher values. Importantly, the location of the activation foci (MPFC, mean Talairach coordinates: $x$, $0 \pm 3 ; y, 48 \pm 10 ; z, 20 \pm 9$, mean volume: $2000 \pm 1200 \mathrm{~mm}^{3}$, striatum, mean Talairach coordinates: $x, 1 \pm 10 ; y, 7 \pm 4 ; z$, $8 \pm 7$, mean volume: $900 \pm 1000 \mathrm{~mm}^{3}$ ) was similar to that reported in previous studies in our lab for subjective value in the context of choice (Kable and Glimcher, 2007; Levy et al., 2010).

Viewing of goods in the scanner in the absence of choice

Subjects viewed images of 20 different goods (CDs, DVDs, books, posters, stationary items, and 5 monetary lotteries) in the scanner (Fig. 3a). Each item was viewed 12 times. To maintain subject alertness, on a few random trials (one repetition of each item) they were asked to choose between the presented item and an unpredictable amount of money. One of these trials was randomly selected at the end of the experiment and subjects received their choice on that trial. Those few within-scanner question trials were excluded from further analysis. Subjects were not told that they would later perform a choice task outside of the scanner.

\section{Choices outside the scanner}

Following removal from the scanner subjects were asked to perform a choice task, in which each item they had seen in the scanner was paired with all other items, and each pair was repeated twice. At the end of the experiment one trial from the choice task was also randomly selected and subjects were given the item they had chosen on that trial. Subjects were mostly consistent in their choices, making the same choice in repetitions of the same pair $(90 \pm 1 \% \mathrm{SD})$, and largely maintaining transitivity $(96 \pm 2 \%$ transitive triplets, i.e., triplets in which if item A was preferred to item B and item B was preferred to item $\mathrm{C}$, item A was also preferred to item C). To verify that the random amounts of money used in the question trials in the scanner did 
not bias subjects' choices outside of the scanner we computed the correlation between the amounts assigned to the different items and the number of times each item was chosen. There was no significant correlation between the amount of money assigned to an item and the number of times it was chosen. In fact, there was a trend toward a correlation in the opposite direction, such that items that were assigned higher amounts in the scanner were chosen slightly less outside of the scanner $(r=-0.36, p=0.06, n=20)$. Since the lottery items were randomly assigned low amounts, but were often chosen, we repeated the correlation calculation without the lotteries, and obtained a completely insignificant level of correlation $(r=-0.16, p=0.3, n=15)$ demonstrating that the price an item was offered at did not affect subsequent choice. The choices made by each subject were used to infer his or her preference ranking for the items. These rankings were highly idiosyncratic across subjects (supplemental Fig. 1, available at www.jneurosci. org as supplemental material) such that the individual preferences of a given subject could not be predicted from preferences exhibited by other subjects (mean correlation of ranking between pairs of subjects, excluding lotteries: $r=0.1 \pm 0.3$ ).

\section{Regression-based analysis}

We first searched globally for brain areas whose BOLD activation during the passive viewing was correlated with the preference ranking inferred from the choices made outside of the scanner. A random-effects group analysis ( $n=12, p<0.05$, FDR corrected) revealed significant correlation in regions of the striatum, MPFC, PCC, posterior superior temporal sulcus extending to the intraparietal sulcus, middle frontal gyrus, and inferior frontal gyrus (supplemental Fig. 2, available at www.jneurosci.org as supplemental material), all areas that have been implicated before in the representation of subjective value with (Hsu et al., 2005; Huettel et al., 2006; Kable and Glimcher, 2007; Tom et al., 2007; Levy et al., 2010) and without (O'Doherty et al., 2002; O'Doherty, 2004; Knutson et al., 2005) choice. We next turned to the ROI analysis to directly test whether activity measured from independently localized areas is correlated with subsequent choices.

\section{Goods activation in ROIs}

Using the localizer task we identified ROIs in the MPFC and the striatum within each subject (Fig. 2) and sampled their activation during the passive viewing of goods. Repeated responses to each item were then averaged within each ROI within each subject, excluding those trials in which subjects were asked to make a choice between the item and an amount of money. The mean BOLD responses were then correlated with the preference ranking computed as the number of times each item was chosen in the choice task (Fig. 4). Positive correlations were obtained in most subjects (9/12 in the MPFC and 9/11 in the striatum) and a Fisher transformation showed that the level of correlation was significant across subjects (MPFC: $r=0.18, p<0.05$; striatum: $r=$ $0.18, p<0.01)$. Significant correlation was also found when choices from all subjects were aggregated (MPFC: $r=0.17, p<$ 0.01 ; striatum: $r=0.16, p<0.05)$. Similarly, significant correla-
MPFC activity and preference ranking
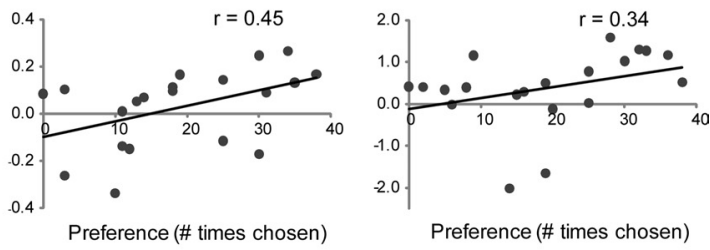

Striatum activity and preference ranking

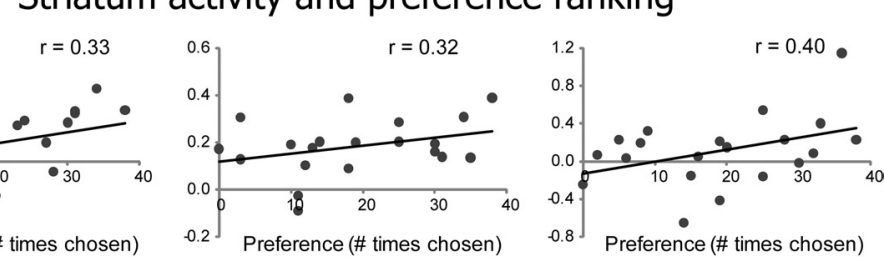

S1

S2

S3 same ranking was averaged across subjects and correlated with the ranking (MPFC: $r=0.49, p<0.05$; striatum: $r=0.50, p<$ 0.05; supplemental Fig. 3, available at www.jneurosci.org as supplemental material).

The observed correlations might have been a result of a general arousal effect, in which more preferred items elicited higher arousal and therefore higher general brain activation. To test whether the effect was specific to these hypothesized valuerelated areas we therefore used the localizer to define an additional ROI in the occipital cortex (in the vicinity of the primary visual cortex). Note that only voxels that showed significantly higher activation for wins compared to losses in the localizer task were included in the definition of the occipital cortex ROI. Therefore, if the effects we observed in the MPFC and the striatum were due to a general arousal effect occurring throughout the brain, we should expect a similar effect in the occipital ROI. This was not the case: the correlation in this ROI was near zero $(r=0.07, p=$ 0.7, supplemental Fig. 3, available at www.jneurosci.org as supplemental material). Although it is still possible that BOLD activation from other, non-value-related, areas is correlated with subsequent choices due to some sort of arousal effect, it is clear that those choices could not be predicted based on a general arousal effect present throughout the brain.

\section{Predicting choice}

To explicitly predict choice we used the activation sampled from the value-related ROIs in each individual subject. Mean responses to the repetitions of each item were averaged and items were ordered according to the level of neural activation. Predictions of choices in pairwise comparisons were then made based on a comparison of the responses to the different items. Figure 5 presents the percentages of correct predictions as a function of the ordinal distance between the neural responses. As can be seen, when activations are most distant, the percentages of correct predictions are high both in the MPFC and in the striatum, with $83 \%$ and $82 \%$ correct predictions, respectively, for the pairs that yielded the most different activation in each subject. As activations became closer in ordinal distance, the percentages of correct predictions decreased, but remained high for all pairs with an activation rank distance of eight or more. Conversely, in the oc- 

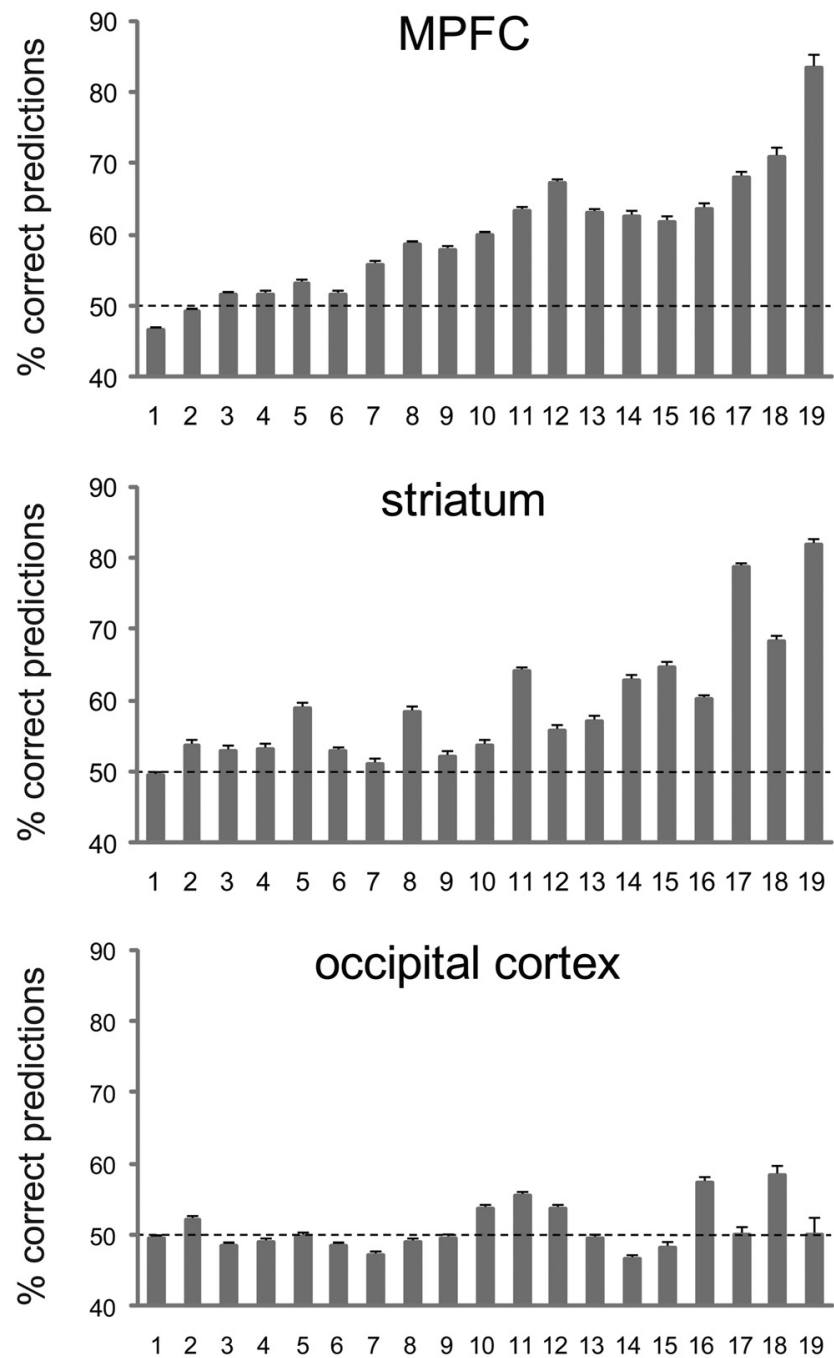

\section{Ordinal distance in BOLD reponse}

Figure 5. Choice predictions based on activation from the predefined ROls. Items were ranked according to the amplitude of the BOLD response they gave rise to, and percentages of correct predictions were calculated separately for each ordinal distance. Error bars, binomial SEM across all choices.

cipital cortex prediction levels were around chance level for all activation rank distances. The difference between the areas can be clearly seen when all choices are pooled together (supplemental Fig. 4, available at www.jneurosci.org as supplemental material). The overall prediction rate using activation from the MPFC was $56 \pm 3 \%$ (SEM across subjects), and a similar percentage of correct predictions was achieved using activation from the striatum (55 $\pm 2 \%$ ) and combining the MPFC and the striatal activation $(56 \pm 3 \%)$. These percentages were significantly different from chance in the striatum and in the combined ROI $(p<0.05$, 1 -tailed $t$ test) and close to significance in the MPFC $(p=0.07)$, while the percentage of correct predictions based on occipital activation was not different from chance $(50 \pm 3 \%, p=0.5)$.

Several factors could lead to a discrepancy between how much subjects valued a certain item compared to other items and whether they chose the same item over those other items. For example, subjects may have already owned some of the items, which might be interpreted to mean that they valued them highly, but would never choose them in the choice task. Similarly, other items might have been completely unfamiliar to subjects, in which case ambiguity about the goods might also make an analysis of value in the absence of choice problematic. To assess these complicating factors, at the end of the experiment we asked subjects to indicate for each item whether they owned it and whether they had heard of it before the experiment. We then recalculated the percentages of correct predictions, limiting our predictions to choices between items that were familiar to subjects but not owned by them (supplemental Fig. 4, available at www.jneurosci. org as supplemental material). This had the effect of increasing the accuracy of our predictions slightly (MPFC: $57 \pm 3 \%$; striatum: $58 \pm 3 \%$; combined: $57 \pm 3 \%, p<0.05$ for all ROIs). The percentage of correct predictions based on occipital activation, however, remained at chance level under these conditions (51 \pm $3 \%, p=0.4)$.

One final confounding factor we explored was the possibility that the correct predictions we made were driven mainly by the lotteries, whose ranking might be assumed to be identical across subjects. We therefore recalculated the predictions excluding pairs in which both items were lotteries. The prediction accuracy was almost identical to the original accuracy (supplemental Fig. 4, available at www.jneurosci.org as supplemental material).

\section{Discussion}

Using fMRI we show here that in the absence of active choice (the kind that neoclassical economics posits is the only marker for utility) the activity of two brain areas previously associated with value representations, the MPFC and the striatum, can be used to predict later consumer choices in individual subjects. This is a finding which explicitly lies outside the domain of traditional economic approaches, but which nonetheless can be related to utility through choice.

Neural activations in predefined brain areas were measured while subjects viewed 20 different goods inside the scanner. Importantly, subjects did not make active choices during either the functional localizer or the goods task, nor did they know that they would later be asked to make such choices. The sampled activations were then used to construct an ordinal neural ranking of the 20 items. Subjects were next removed from the scanner and asked to make all possible pairwise choices among the same goods. These choices were used to create an ordinal choice preference ranking of the 20 items. These two sets of rankings, the neural ranking and the behavioral ranking, were significantly correlated in our subjects. Moreover, using the neural ranking to predict each pairwise choice for each subject we found that prediction accuracy increased as a function of the neural rank distance between the objects in the pair, peaking at above $80 \%$ correct for the greatest neural rank distance. Finally, the effect was specific to value-related areas: activation measured from a region in occipital cortex could not be used to predict choice. These results imply that the same "subjective values" (Glimcher, 2009) that can be deduced from choices are also generated in the absence of choice, at least at the level of the BOLD signal, by the same neural mechanisms that are active during choice.

\section{Value-related areas}

Converging evidence suggests that the striatum and MPFC are part of a general valuation system that represents value under many different conditions. Activity in the striatum is correlated with the magnitude of unexpected rewards and punishments (Delgado et al., 2000; Kuhnen and Knutson, 2005), as well as with the amount (Breiter et al., 2001; Knutson et al., 2001a, 2003), the probability (Hsu et al., 2009), the expected value (Hsu et al., 2005; 
Preuschoff et al., 2006; Tobler et al., 2007; Tom et al., 2007; Luhmann et al., 2008) and the marginal utility (Pine et al., 2009) of predicted outcomes, and may even reflect a form of reference dependence (De Martino et al., 2009). Similar findings have been reported for the MPFC, which also responds to both receipt of monetary reward (Knutson et al., 2001b, 2003; Kuhnen and Knutson, 2005) and expected rewards (McClure et al., 2004a), correlating with the expected value of rewards (Knutson et al., 2005). Finally, activity in the striatum and the MPFC has been shown to track the discounted value of future rewards (Kable and Glimcher, 2007) and the decision value of both risky and ambiguous expected rewards (Levy et al., 2010).

Other studies have shown that the striatum and MPFC also represent the value of nonmonetary rewards. The striatum has been shown to respond to the anticipation of primary rewards (O'Doherty et al., 2002; McClure et al., 2007), as well as other nonmonetary rewards (Sharot et al., 2009), and its activity reflects behavioral preferences, such as preferences for different types of juice (O'Doherty et al., 2006) and meal pleasantness ratings (Small et al., 2003). In a similar way, recent studies have reported the overlapping representations of action-value and stimulus-value in the MPFC (Gläscher et al., 2009) as well as an overlapping representation of the value of different types of goods (Chib et al., 2009), and the effect of subjectively weighted decision factors on brain activation (Hare et al., 2009).

\section{The value of consumer goods}

The picture that emerges from all of these studies is of a unified valuation system that represents the value of a wide range of different objects, in different domains and under different conditions. It is therefore reasonable to expect these areas to represent the value of different consumer goods. Indeed, activation in the MPFC has been shown to be stronger for preferred compared to nonpreferred brands of beer and coffee during choice (Deppe et al., 2005), as well as for preferred types of cars in the absence of choice (Erk et al., 2002). In a more recent study by Knutson et al. (2007), subjects made explicit choices in the scanner and these choices were then related to later ratings of the same items made outside of the scanner. In that choice task, subjects first viewed an image of a consumer good, followed by its price, and were then asked to decide whether to purchase the good. Striatal activity during the presentation of the product and MPFC activity during the presentation of the price significantly predicted the subsequent purchase decisions, and striatal activations during choice predicted later ratings of the products. Importantly, however, the Knutson study measured neural activation while subjects were making choices. The MPFC activation was recorded when all the information for making the choice (product and price) was already available to the subjects in a period immediately before they expressed their choices by a key-press. The striatal activation, which predicted later ratings, was recorded when the price was still unknown, but subjects knew that they would view a price and make a choice in a few seconds, such that the activation measurement was still done in the context of choice.

\section{Value in the absence of choice}

A recent study (Lebreton et al., 2009) was the first to directly link activation in the absence of active choice to subsequent choices. Subjects in this study were scanned while rating either the pleasantness or the age of faces, houses and paintings. Once outside of the scanner, subjects were presented with pairs of the same images and asked to identify one of the two images as visually more pleasant. By searching for brain areas that were more active for images that were subsequently preferred compared to images that were not preferred the authors identified regions in the striatum and the MPFC. Interestingly, a similar result was reported in two earlier studies that did not ask this question directly. In the first study (McClure et al., 2004b) activation in the MPFC in response to an unidentified squirt of Coke or Pepsi was correlated with subjects' taste preference as deduced from choices among unlabeled soft drinks made outside the scanner. In the second study (Berns et al., 2008) activation in a vast network of brain areas, including regions in the MPFC and the striatum, during expectation of aversive outcomes predicted subsequent choices between the same outcomes. These results suggest that at least the valuation processes that are involved in pleasantness judgments may indeed take place automatically in these areas, whether or not those valuations are required for the task.

The present study extends these previous results by showing that activation in the valuation areas in the absence of choice is also correlated with more complex, multidimensional valuations. A consumer choice takes into account many factors, some of which may have opposing effects on the final decision (e.g., a pleasant music CD with a visually unpleasant cover image or vice versa). Moreover, a consumer choice carries practical consequences for the decision-maker that a mere attractiveness preference judgment does not. Our results suggest that automatic valuations take place even in the absence of choice, and that the same neural mechanisms are engaged by this representation whether or not choice is made.

Finally, it should be noted that although previous studies have clearly shown that areas in the MPFC and the striatum represent the value of both experienced and anticipated outcomes, it is still possible that these areas do not completely overlap. If this is the case, then the accuracy of our choice predictions might slightly improve if activity is sampled from the areas that represent the value of anticipated, rather than experienced, outcomes, or if a whole brain-based classifier analysis were performed. At the same time, the drop in prediction accuracy, from $>80 \%$ for the two goods with the most divergent neural representations in the localized value-areas, to close to chance level for items with more similar, although still distinct, neural activation profiles, suggests fairly stringent bounds on the claims of neuromarketers.

\section{References}

Berns GS, Capra CM, Chappelow J, Moore S, Noussair C (2008) Nonlinear neurobiological probability weighting functions for aversive outcomes. Neuroimage 39:2047-2057.

Boynton GM, Engel SA, Glover GH, Heeger DJ (1996) Linear systems analysis of functional magnetic resonance imaging in human V1. J Neurosci 16:4207-4221.

Breiter HC, Aharon I, Kahneman D, Dale A, Shizgal P (2001) Functional imaging of neural responses to expectancy and experience of monetary gains and losses. Neuron 30:619-639.

Caplin A, Dean M, Glimcher PW, Rutledge RB (2010) Measuring beliefs and rewards: a neuroeconomic approach. Q J Econ 125:923-960.

Chib VS, Rangel A, Shimojo S, O’Doherty JP (2009) Evidence for a common representation of decision values for dissimilar goods in human ventromedial prefrontal cortex. J Neurosci 29:12315-12320.

Delgado MR, Nystrom LE, Fissell C, Noll DC, Fiez JA (2000) Tracking the hemodynamic responses to reward and punishment in the striatum. J Neurophysiol 84:3072-3077.

De Martino B, Kumaran D, Holt B, Dolan RJ (2009) The neurobiology of reference-dependent value computation. J Neurosci 29:3833-3842.

Deppe M, Schwindt W, Kugel H, Plassmann H, Kenning P (2005) Nonlinear responses within the medial prefrontal cortex reveal when specific implicit information influences economic decision making. J Neuroimaging 15:171-182. 
Erk S, Spitzer M, Wunderlich AP, Galley L, Walter H (2002) Cultural objects modulate reward circuitry. Neuroreport 13:2499-2503.

Friston J, Homes A, Worsley K, Poline J, Frith C, Frackwowiak R (1995) Statistical parametric maps in functional imaging: a general linear approach. Hum Brain Mapp 2:189-210.

Genovese CR, Lazar NA, Nichols T (2002) Thresholding of statistical maps in functional neuroimaging using the false discovery rate. Neuroimage 15:870-878.

Gläscher J, Hampton AN, O’Doherty JP (2009) Determining a role for ventromedial prefrontal cortex in encoding action-based value signals during reward-related decision making. Cereb Cortex 19:483-495.

Glimcher PW (2009) Choice: towards a standard back-pocket model. In: Neuroeconomics: decision making and the brain (Glimcher PW, Camerer CF, Fehr E, Poldrack RA, eds), pp 503-521. New York: Academic.

Hare TA, Camerer CF, Rangel A (2009) Self-control in decision-making involves modulation of the vmPFC valuation system. Science $324: 646-$ 648.

Haynes JD, Rees G (2006) Decoding mental states from brain activity in humans. Nat Rev Neurosci 7:523-534.

Hsu M, Bhatt M, Adolphs R, Tranel D, Camerer CF (2005) Neural systems responding to degrees of uncertainty in human decision-making. Science 310:1680-1683.

Hsu M, Krajbich I, Zhao C, Camerer CF (2009) Neural response to reward anticipation under risk is nonlinear in probabilities. J Neurosci 29:2231-2237.

Huettel SA, Stowe CJ, Gordon EM, Warner BT, Platt ML (2006) Neural signatures of economic preferences for risk and ambiguity. Neuron 49:765-775.

Kable JW, Glimcher PW (2007) The neural correlates of subjective value during intertemporal choice. Nat Neurosci 10:1625-1633.

Kable JW, Glimcher PW (2009) The neurobiology of decision: consensus and controversy. Neuron 63:733-745.

Knutson B, Adams CM, Fong GW, Hommer D (2001a) Anticipation of increasing monetary reward selectively recruits nucleus accumbens. J Neurosci 21:RC159(1-5).

Knutson B, Fong GW, Adams CM, Varner JL, Hommer D (2001b) Dissociation of reward anticipation and outcome with event-related fMRI. Neuroreport 12:3683-3687.

Knutson B, Fong GW, Bennett SM, Adams CM, Hommer D (2003) A region of mesial prefrontal cortex tracks monetarily rewarding outcomes: characterization with rapid event-related fMRI. Neuroimage 18:263-272.

Knutson B, Taylor J, Kaufman M, Peterson R, Glover G (2005) Distributed neural representation of expected value. J Neurosci 25:4806-4812.

Knutson B, Rick S, Wimmer GE, Prelec D, Loewenstein G (2007) Neural predictors of purchases. Neuron 53:147-156.

Kuhnen CM, Knutson B (2005) The neural basis of financial risk taking. Neuron 47:763-770.

Lebreton M, Jorge S, Michel V, Thirion B, Pessiglione M (2009) An automatic valuation system in the human brain: evidence from functional neuroimaging. Neuron 64:431-439.

Levy I, Snell J, Nelson AJ, Rustichini A, Glimcher PW (2010) Neural repre- sentation of subjective value under risk and ambiguity. J Neurophysiol 103:1036-1047.

Luhmann CC, Chun MM, Yi DJ, Lee D, Wang XJ (2008) Neural dissociation of delay and uncertainty in intertemporal choice. J Neurosci 28:14459-14466.

McClure SM, Laibson DI, Loewenstein G, Cohen JD (2004a) Separate neural systems value immediate and delayed monetary rewards. Science 306:503-507.

McClure SM, Li J, Tomlin D, Cypert KS, Montague LM, Montague PR (2004b) Neural correlates of behavioral preference for culturally familiar drinks. Neuron 44:379-387.

McClure SM, Ericson KM, Laibson DI, Loewenstein G, Cohen JD (2007) Time discounting for primary rewards. J Neurosci 27:5796-5804.

O’Doherty JP (2004) Reward representations and reward-related learning in the human brain: insights from neuroimaging. Curr Opin Neurobiol 14:769-776.

O’Doherty JP, Deichmann R, Critchley HD, Dolan RJ (2002) Neural responses during anticipation of a primary taste reward. Neuron 33:815-826.

O’Doherty JP, Buchanan TW, Seymour B, Dolan RJ (2006) Predictive neural coding of reward preference involves dissociable responses in human ventral midbrain and ventral striatum. Neuron 49:157-166.

Pine A, Seymour B, Roiser JP, Bossaerts P, Friston KJ, Curran HV, Dolan RJ (2009) Encoding of marginal utility across time in the human brain. J Neurosci 29:9575-9581.

Preuschoff K, Bossaerts P, Quartz SR (2006) Neural differentiation of expected reward and risk in human subcortical structures. Neuron 51:381-390.

Samuelson PA (1938) A note on the pure theory of consumer behavior. Economia 1:61-71.

Schultz W (2009) Midbrain dopamine neurons: a retina of the reward system? In: Neuroeconomics: decision making and the brain (Glimcher PW, Camerer CF, Fehr E, Poldrack RA, eds), pp 323-329. New York: Academic.

Schultz W, Dayan P, Montague PR (1997) A neural substrate of prediction and reward. Science 275:1593-1599.

Sharot T, De Martino B, Dolan RJ (2009) How choice reveals and shapes expected hedonic outcome. J Neurosci 29:3760-3765.

Small DM, Jones-Gotman M, Dagher A (2003) Feeding-induced dopamine release in dorsal striatum correlates with meal pleasantness ratings in healthy human volunteers. Neuroimage 19:1709-1715.

Talairach J, Tournoux P (1988) Co-planar stereotaxic atlas of the human brain. New York: Thieme Medical Publishers.

Tobler PN, O’Doherty JP, Dolan RJ, Schultz W (2006) Human neural learning depends on reward prediction errors in the blocking paradigm. J Neurophysiol 95:301-310.

Tobler PN, O’Doherty JP, Dolan RJ, Schultz W (2007) Reward value coding distinct from risk attitude-related uncertainty coding in human reward systems. J Neurophysiol 97:1621-1632.

Tom SM, Fox CR, Trepel C, Poldrack RA (2007) The neural basis of loss aversion in decision-making under risk. Science 315:515-518. 